\title{
The Improvement of DS Evidence Theory and Its Application in IR/MMW Target Recognition
}

\author{
Yibing Li, ${ }^{1}$ Jie Chen, ${ }^{1}$ Fang Ye, ${ }^{1}$ and Dandan Liu ${ }^{2}$ \\ ${ }^{1}$ College of Information and Communication Engineering, Harbin Engineering University, Harbin 150001, China \\ ${ }^{2}$ College of Electrical and Control Engineering, Heilongjiang University of Science and Technology, Harbin 150001, China \\ Correspondence should be addressed to Fang Ye; yefang0815@sina.cn
}

Received 4 July 2015; Revised 11 October 2015; Accepted 18 October 2015

Academic Editor: Stephane Evoy

Copyright (c) 2016 Yibing Li et al. This is an open access article distributed under the Creative Commons Attribution License, which permits unrestricted use, distribution, and reproduction in any medium, provided the original work is properly cited.

\begin{abstract}
ATR system has a broad application prospect in the military field, especially in the field of modern defense technology. When paradoxes are in existence in ATR system due to adverse battlefield environment, integration cannot be effectively and reliably carried out only by traditional DS evidence theory. In this paper, a modified DS evidence theory is presented and applied in IR/MMW target recognition system. The improvement of DS evidence theory is realized by three parts: the introduction of sensor priority and evidence credibility to realize the discount processing of evidences, the modification of DS combination rule to enhance the accuracy of synthesis results, and the compound decision-making rule. The application of the modified algorithm in IR/MMW system is designed to deal with paradoxes, improve the target recognition rate, and ensure the reliability of target recognition system. Experiments are given to illustrate that the introduction of the modified DS evidence theory in IR/MMW system is better able to realize satisfactory target recognition performance through multisensor information fusion than any single-mode system.
\end{abstract}

\section{Introduction}

Automatic target recognition system, namely, the ATR system, has become an important part of the present and the future weapon system. For ATR systems in the modern warfare, with the growing complexity of battlefield environment, information acquired from sensors is often incomplete and inaccurate and has some degree of uncertainty and fuzziness, possibly even contradictory. ATR systems with single sensor have many limitations like weak anti-interference ability and low recognition reliability. Single-mode systems cannot suit the demand of future battlefield because of their dependence on the observation environment. The complementarity and synergistic interaction of sensors can improve the ATR ability in dynamic scene. In consideration of defects and limitations of single-mode systems, IR/MMW fusion, as an inevitable trend of compound target recognition algorithm, can adopt others' strong points while overcoming its weak point and make the system adapt to continued deterioration of battlefield environment and dynamical changes of objectives.

Uncertainty reasoning is the foundation of IR/MMW system, which can deal with incomplete, uncertain, and unclear information that exists in IR/MMW system effectively. DS evidence theory, also known as Dempster-Shafer theory, is common and widely used uncertainty reasoning $[1,2]$. It has been widely used in many fields like expert system [3, $4]$, artificial intelligence $[5,6]$, fault diagnosis $[7,8]$, target recognition $[9,10]$, target tracking [11], decision-making [12], information fusion [13], and so forth. However, some researches found that traditional DS evidence theory cannot produce reasonable synthesis results in the case of paradoxes (because of the complexity of practical environment and probable conflicts of evidence sources) [14]. Therefore, DS evidence theory is improved primarily before its application in IR/MMW system.

Domestic and foreign researchers have done a lot of researches to solve paradoxes, which are mainly divided into two categories: the improved DS combination rules and the modified conflict evidences methods.

Some researchers think that unreasonable results are mainly caused by the normalization step of DS combination rule. Thus, it optimizes the DS combination rule by giving evidence conflicts to certain subset with certain proportion, which is called the improved DS combination rule. In Smets's 
opinion, $\Theta$ is regarded as an incomplete set and conflicts are given to an unknown proposition [15]. This method solves paradoxes theoretically, but it actually increases the uncertainty of synthesis results by introducing an unknown proposition. Yager allotted conflicts directly to $\Theta[16]$. However, it can only settle paradoxes efficiently with two evidence sources in system, and it is too conservative to admit the useful information that exists in conflict evidences. On this basis, an improved method is proposed by dividing evidences into support evidences and conflict evidences, which solves the problem of unequal information quantity among evidences [17]. In addition, another algorithm proportionally allocates the conflicts to every focal element of conflict evidences through the introduction of a weight factor that is proportional to the conflicts [18]. In order to distinguish the local conflicts and global conflicts effectively and make the system robust simultaneously, a new DS combination rule named absorptive method is put forward by allocating local conflicts directly to local propositions [19].

Other scholars consider that paradoxes are mainly caused by unreliable evidences. Therefore, this kind of method modifies evidences before evidences combine instead of changing the DS combination rule, which is called the modified conflict evidences method. Among them, an improved method is put forward by considering the average mean of evidences as a new evidence before evidences combination [20]. But it is obvious that the idea only averages the evidences without considering their differences. Thus, Horiuchi came up with a method through calculating a new evidence by the weight sum of evidences instead of simple average [21]. In order to efficiently combine high conflict evidences, a novel method is proposed through the introduction of the distance function introduced by Lefevre, which can improve the reliability of system [21]. To manage conflict evidences, global conflicts are allocated in detail to several local conflicts, and a new method is presented based on number, reliability, and relevance of evidence sources [22].

These two mainstream improved methods solve paradoxes just on the sight of single angle without fully considering the differences among sensors and uncertainty of observation environment. In this paper, the novel algorithm firstly takes the consistency and reliability of evidence sources into consideration concurrently and thus introduces sensor priority and evidence credibility to realize discount processing of evidences. Then, it cancels the normalization step of DS combination rule to decrease the interference and unpredictability of observation environment and further enhance the reliability and rationality of synthesis results. Finally, it puts the synthesis results into decision-making rule to get target recognition results. Experiment results demonstrate that the application of the novel method in IR/MMW system can improve the target recognition rate, ensure reliable operation of system, and enhance the battlefield adaptability, antijamming immunity, antistealth performance, and precision of target identification.

This paper is organized as follows. The foundation and discussions of traditional DS evidence theory are summarized in Section 2. Then, as the cores of this paper, Section 3 highlights the modified DS evidence theory, and Section 4 provides the specific implementation steps of its application in IR/MMW target recognition system. In Section 5, experiment results and analyses are shown to manifest the validity of the novel algorithm from the points of theory and application. And conclusions are presented in Section 6 at the end.

\section{DS Evidence Theory}

Researches were done for ATR system using DS evidence theory back in the 90s $[23,24]$. The feasibility of applying DS evidence theory in ATR system has been testified in [25]. And in [26], it is shown that, compared with Sugero's theory and possibility theory, the best performance in ATR system is achieved by DS evidence theory. Thus, DS evidence theory makes the system not only more powerful but also more robust [27].

2.1. Preliminaries. DS evidence theory firstly supposes the definition of a finite nonempty set of hypotheses as the frame of discernment (called FoD for short), which consists of $N$ mutually exclusive and exhaustive hypotheses. The FoD is defined as follows:

$$
\Theta=\left\{H_{1}, H_{2}, \ldots, H_{N}\right\},
$$

where $N$ is the number of hypotheses in system and $H$ is a hypothesis of the FoD.

For example, in pattern recognition system, there are a set of $N$ mutually disjoint classes in the pattern space. Thus, the FoD should be defined as $\Theta=\left\{w_{1}, w_{2}, \ldots, w_{N}\right\}$, where $w$ is a class in the pattern space.

Then, let us denote $2^{\Theta}$ as the power set, which is composed of $2^{N}$ propositions of $\Theta$ :

$$
\begin{aligned}
& 2^{\Theta}=\left\{\Phi, H_{1}, H_{2}, \ldots, H_{N},\left\{H_{1} \cup H_{2}\right\},\left\{H_{1} \cup H_{3}\right\}, \ldots,\right. \\
&\left.\left\{H_{1} \cup H_{2} \cup \cdots H_{N}\right\}\right\},
\end{aligned}
$$

where $\Phi$ is the empty set and any proposition in $2^{\Theta}$ is a subset of $\Theta$.

The basic probability assignment (called BPA for short) on $2^{\Theta}$, also called the basic belief assignment (called BBA for short), is a function $m: 2^{\Theta} \rightarrow[0,1]$, which should satisfy the following conditions:

$$
\begin{aligned}
m(\Phi) & =0 \\
\sum_{A \subseteq \Theta} m(A) & =1,
\end{aligned}
$$

where $A$ is a proposition in $2^{\Theta}$ which contains one or more hypotheses and $m(A)$ represents the initial support degree for proposition $A$.

Due to the lack of further information, the BPA of proposition $A$ cannot be subdivided into its proper subsets. Any proposition $A$ satisfying $m(A)>0(A \subseteq \Theta)$ is called a focal element, and the set of all focal elements is called the core of BPA. 


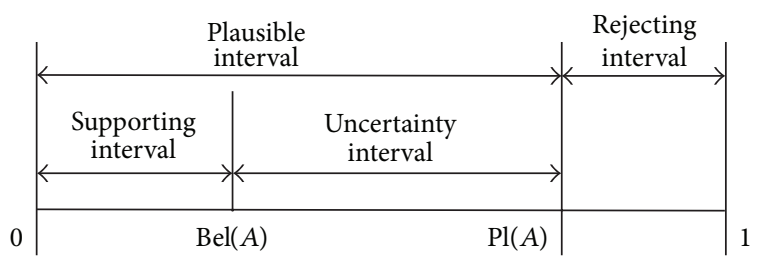

FIgure 1: The relationship diagram of $\operatorname{Bel}(A)$ and $\operatorname{Pl}(A)$.

The belief function (called Bel for short) and plausibility function (called Pl for short) in $2^{\Theta}$ are defined, respectively, as

$$
\begin{aligned}
\operatorname{Bel}(A) & =\sum_{B \subseteq A} m(B) \\
\operatorname{Pl}(A) & =\sum_{B \cap A \neq \Phi} m(B)
\end{aligned}
$$

$$
A, B \subseteq \Theta,
$$

where $A$ and $B$ are both the propositions in $2^{\Theta}$.

It can be seen from formula (4) that Bel of proposition $A$ is interpreted as the minimum uncertainty value of $A$ which constitutes a lower limit function on the probability of $A$, while its $\mathrm{Pl}$ is interpreted as the maximum uncertainty value of $A$ which constitutes an upper limit function. And the relationship between $\operatorname{Bel}(A)$ and $\operatorname{Pl}(A)$ is defined as follows, which is shown in Figure 1:

$$
\begin{aligned}
\operatorname{Bel} A & \leq \operatorname{Pl}(A) \\
\operatorname{Pl}(A) & =1-\operatorname{Bel}(\bar{A})
\end{aligned}
$$

$$
A \subseteq \Theta,
$$

where $\bar{A}$ is the complement set of $A$.

The interval $[\operatorname{Bel}(A), \mathrm{Pl}(A)]$ is named the belief interval or uncertainty interval, which represents the uncertainty and imprecision of DS evidence theory. Suppose that $m_{1}, m_{2}, \ldots, m_{N}$ are $N$ mutually independent BPAs from $N$ different sensors in the same FoD based on information detection. The DS combination rule, denoted by $m=m_{1} \oplus$ $m_{2} \oplus \cdots \oplus m_{N}$, is also called the orthogonal sum of evidences. Thus, the combination of $m_{i}, m_{j}(i, j=1,2, \ldots, N)$ can be defined as

$$
\begin{aligned}
m_{i j}(A) & =\frac{1}{1-k} \sum_{A_{i} \cap A_{j}=A} m_{i}\left(A_{i}\right) \cdot m_{j}\left(A_{j}\right) \\
m(\Phi) & =0
\end{aligned}
$$

$$
A \subseteq \Theta, A \neq \Phi,
$$

where $k$ is called the total conflict factor and it represents the total conflicts between evidences $m_{i}$ and $m_{j}$ :

$$
k=\sum_{A_{i} \cap A_{j}=\Phi} m_{i}\left(A_{i}\right) \cdot m_{j}\left(A_{j}\right) .
$$

$k$ demonstrates the degree of conflicts between evidences, and $1 /(1-k)$ is the normalization factor which ensures that the sum of BPAs can be unit, and the BPA for null set is none. According to similar principle, we can calculate corresponding $\operatorname{Bel}(A)$ and $\operatorname{Pl}(A)$.

Obviously, the DS combination rule satisfies both commutative law and associate law, which are shown separately as

$$
\begin{gathered}
m_{1} \oplus m_{2}=m_{2} \oplus m_{1} \\
\left(m_{1} \oplus m_{2}\right) \oplus m_{3}=m_{1} \oplus\left(m_{2} \oplus m_{3}\right) .
\end{gathered}
$$

2.2. Common Paradoxes. Uncertainty of system can be categorized into four areas shown in [28]. Firstly, knowledge representation can be defined as confirmed, probable, possible, doubtful, and improbable to support the propositions, but the system cannot guarantee its objectivity. Secondly, algorithms decide how the uncertainty model performs operations with information, which greatly affect uncertainty reduction. Thirdly, evidence representation usually depends on human experience, which has large invalidity. Lastly, the evidence sources are sensors that exist in environment with a variety of noises.

In this paper, due to the scarce knowledge of observation environment, the system may import a lot of interference and clutter. And the limitation of sensor precision will ulteriorly cause uncertainty and imprecision in DS reasoning. Thus, the paradox research is the premise to analyze the defects of DS evidence theory and realize its improvement.

The common paradoxes are divided into four classes: complete conflict paradox, 0 trust paradox (one ballot veto), 1 trust paradox, and high conflict paradox.

2.2.1. Complete Conflict Paradox. Assuming that the FoD of system is $\Theta=\{A, B, C\}$ and there are two sources of evidence, the BPAs are presented, respectively, as

$$
\begin{aligned}
& m_{1}(A)=1, \\
& m_{1}(B)=0, \\
& m_{1}(C)=0 \\
& m_{2}(A)=0, \\
& m_{2}(B)=1, \\
& m_{2}(C)=0 .
\end{aligned}
$$

According to formula (7), the total conflict factor $k$ can be calculated as $k=1$. It is apparent that evidences $m_{1}$ and $m_{2}$ conflict completely, which causes the denominator of formula (6) to become zero. Under such circumstances, DS combination rule is unable to synthesize. If there are two more sources of evidence, whose BPAs are shown as

$$
\begin{aligned}
& m_{3}(A)=0.8, \\
& m_{3}(B)=0.1, \\
& m_{3}(C)=0.1 \\
& m_{4}(A)=0.8,
\end{aligned}
$$




$$
\begin{aligned}
& m_{4}(B)=0.1, \\
& m_{4}(C)=0.1,
\end{aligned}
$$

from intuitive judgment, the accurate synthesis results should drastically support proposition $A$ because evidences $m_{1}, m_{3}$, and $m_{4}$ all support proposition $A$ with large BPAs. But DS combination rule cannot normally be used when $k=1$. This kind of illogical situation is called complete conflict paradox.

2.2.2. 0 Trust Paradox. Assume that FoD of the system is $\Theta=$ $\{A, B, C\}$, and four BPAs of evidences are

$$
\begin{aligned}
& m_{1}(A)=0.5, \\
& m_{1}(B)=0.2, \\
& m_{1}(C)=0.3 \\
& m_{2}(A)=0, \\
& m_{2}(B)=0.9, \\
& m_{2}(C)=0.1 \\
& m_{3}(A)=0.5, \\
& m_{3}(B)=0.2, \\
& m_{3}(C)=0.3 \\
& m_{4}(A)=0.5, \\
& m_{4}(B)=0.2, \\
& m_{4}(C)=0.3 .
\end{aligned}
$$

The total conflict factor can be calculated as $k=0.99$ in the same way as shown before. Applying formula (7), the synthesis results are

$$
\begin{aligned}
& m(A)=0, \\
& m(B)=0.727, \\
& m(C)=0.273 .
\end{aligned}
$$

It can be checked that because evidence $m_{2}$ totally denies proposition $A$, the BPA for proposition $A$ in the synthesis results will always be zero no matter how strongly evidences $m_{1}, m_{3}$, and $m_{4}$ support proposition $A$. That is, DS combination rule has the disadvantage of one ballot veto.

2.2.3. 1 Trust Paradox. Assume that the FoD of system is $\Theta=$ $\{A, B, C\}$, and there are four evidences. The BPAs are

$$
\begin{aligned}
& m_{1}(A)=0.9 \\
& m_{1}(B)=0.1, \\
& m_{1}(C)=0 \\
& m_{2}(A)=0, \\
& m_{2}(B)=0.1,
\end{aligned}
$$

$$
\begin{aligned}
& m_{2}(C)=0.9 \\
& m_{3}(A)=0.1, \\
& m_{3}(B)=0.15, \\
& m_{3}(C)=0.75 \\
& m_{4}(A)=0.1, \\
& m_{4}(B)=0.15, \\
& m_{4}(C)=0.75 .
\end{aligned}
$$

The total conflict factor can be calculated as $k=0.9998$ and the synthesis results are

$$
\begin{aligned}
& m(A)=0, \\
& m(B)=1, \\
& m(C)=0 .
\end{aligned}
$$

Although all sources of evidence give proposition $B$ small BPAs, the synthesis results completely believe proposition $B$ is the correct proposition, which is perverse in practical application.

2.2.4. High Conflict Paradox. Assume that the FoD of system is $\Theta=\{A, B, C, D, E\}$, and there are five evidences. The BPAs and synthesis results are, respectively, presented in formula (15) and formula (16).

The total conflict factor can be calculated as $k=0.9999$. It can be proved in a similar way that precise synthesis results should support proposition $A$ as evidences $m_{1}, m_{3}, m_{4}$, and $m_{5}$ all give proposition $A$ large BPAs. But high conflicts among evidences actually lead to error reasoning shown in formula (16). Hence,

$$
\begin{aligned}
& m_{1}(A)=0.7, \\
& m_{1}(B)=0.1, \\
& m_{1}(C)=0.1, \\
& m_{1}(D)=0, \\
& m_{1}(E)=0.1 \\
& m_{2}(A)=0 \\
& m_{2}(B)=0.5, \\
& m_{2}(C)=0.2, \\
& m_{2}(D)=0.1, \\
& m_{2}(E)=0.2 \\
& m_{3}(A)=0.6, \\
& m_{3}(B)=0.1, \\
& m_{3}(C)=0.15 \\
& m_{3}(D)=0
\end{aligned}
$$




$$
\begin{aligned}
& m_{3}(E)=0.15 \\
& m_{4}(A)=0.55, \\
& m_{4}(B)=0.1, \\
& m_{4}(C)=0.1, \\
& m_{4}(D)=0.15, \\
& m_{4}(E)=0.1 \\
& m_{5}(A)=0.6, \\
& m_{5}(B)=0.1, \\
& m_{5}(C)=0.2, \\
& m_{5}(D)=0, \\
& m_{5}(E)=0.1 \\
& m(A)=0, \\
& m(B)=0.3571, \\
& m(C)=0.4286, \\
& m(D)=0, \\
& m(E)=0.2143 .
\end{aligned}
$$

\subsection{Relationship among Bayesian Theory, DS Evidence Theory,} and DSmT Reasoning. Relative to probability theory and Bayesian theory, DS evidence theory can settle imprecise information in the absence of prior knowledge and can be viewed as a generalization of probability theory. It can be seen in the previous section that DS evidence theory cannot handle the information with paradoxes. In order to overcome the problem, Jean Dezert and Florentin Smarandache put forward the DSmT reasoning which can combine uncertain, imprecise, and contradictory information [29]. DSmT reasoning is an extension of traditional DS evidence theory, and it is widely applied in edge detection in color images [30], aircraft recognition [31], remote sensing image classification [32], and so forth.

In 2003, Jean Dezert presents the hyperpower set notation as $D^{\Theta}$ for DSmT. It retains the contradictory focal elements, while greatly increasing the total number of focal elements. Examples are given as follows to show the increasing calculational complexity of DSmT compared with DS evidence theory:

(1) $\Theta=\{\}\left(\right.$ empty): $2^{\Theta}=\{\Phi\}, D^{\Theta}=\{\Phi\}$.

(2) $\Theta=\left\{H_{1}\right\}: 2^{\Theta}=\left\{\Phi, H_{1}\right\}, D^{\Theta}=\left\{\Phi, H_{1}\right\}$.

(3) $\Theta=\left\{H_{1}, H_{2}\right\}: 2^{\Theta}=\left\{\Phi, H_{1}, H_{2}, H_{1} \cup H_{2}\right\}, D^{\Theta}=$ $\left\{\Phi, H_{1}, H_{2}, H_{1} \cup H_{2}, H_{1} \cap H_{2}\right\}$.

(4) $\Theta=\left\{H_{1}, H_{2}, H_{3}\right\}: 2^{\Theta}=\left\{\Phi, H_{1}, H_{2}, H_{3}, H_{1} \cup H_{2}, H_{1} \cup\right.$ $\left.H_{3}, H_{2} \cup H_{3}, H_{1} \cup H_{2} \cup H_{3}\right\}, D^{\Theta}=\left\{\Phi, H_{1}, H_{2}, H_{3}, H_{1} \cup\right.$ $H_{2}, H_{1} \cup H_{3}, H_{2} \cup H_{3}, H_{1} \cap H_{2}, H_{1} \cap H_{3}, H_{2} \cap H_{3}, H_{1} \cup$

$$
\begin{aligned}
& H_{2} \cup H_{3}, H_{1} \cap H_{2} \cap H_{3},\left(H_{1} \cup H_{2}\right) \cap H_{3},\left(H_{1} \cup H_{3}\right) \cap \\
& H_{2},\left(H_{2} \cup H_{3}\right) \cap H_{1},\left(H_{1} \cap H_{2}\right) \cup H_{3},\left(H_{1} \cap H_{3}\right) \cup H_{2},\left(H_{2} \cap\right. \\
& \left.\left.H_{3}\right) \cup H_{1},\left(H_{1} \cup H_{2}\right) \cap\left(H_{1} \cap H_{3}\right) \cap\left(H_{2} \cup H_{3}\right)\right\} .
\end{aligned}
$$

From these discussions, it is obvious that DS evidence theory is an extension of Bayesian theory, and DSmT reasoning is viewed as a general flexible approach for managing uncertainty and conflicts for a wide class of fusion problems where the information to combine is modeled as a finite set of belief functions provided by different independent sources $[33,34]$. And these three algorithms are used in similar applications. It is easy to check that, with the increasing number of FoD, the gap on the calculational amount between DS evidence theory and DSmT reasoning becomes larger. Thus, considering DSmT's large computational burden, this paper uses DS evidence theory as the uncertain reasoning method in the IR/MMW target recognition system, which can not only ensure the identification speed of system, but also build the basis for DSmT's application implementation.

2.4. Comparison between Two Mainstream Improved Methods and PCR5. In the DSmT reasoning's FoD, proportional conflict redistribution 5 (called PCR5 for short) is used generally to combine the BPAs [33]. PCR5 transfers the conflict mass only to the elements involved in the conflict and proportionally to their individual masses, which is similar to the way of assigning local conflicts directly to local propositions [19]. As PCR5 can be used in DS evidence theory as well, we take two mainstream improved methods to give a simple comparison.

The PCR5 rule in DS evidence theory is defined as follows (assuming that there are two evidences):

$$
m(A)=\sum_{A_{1} \cap A_{2}=A} m_{1}\left(A_{1}\right) \cdot m_{2}\left(A_{2}\right)+\Delta\left(A, 2^{\Theta}\right),
$$

where $\Delta\left(A, 2^{\Theta}\right)=\sum_{A_{3} \cap A=\Phi}\left[m_{1}(A)^{2} \cdot m_{2}\left(A_{3}\right) /\left(m_{1}(A)+\right.\right.$ $\left.\left.m_{2}\left(A_{3}\right)\right)+m_{2}(A)^{2} \cdot m_{1}\left(A_{3}\right) /\left(m_{2}(A)+m_{1}\left(A_{3}\right)\right)\right]$ and $A_{1}, A_{2}, A_{3} \subseteq 2^{\Theta}$.

The general description of the improved DS combination rules presented in Section 1 can be shown as

$$
m(A)=\sum_{A_{1} \cap A_{2}=A} m_{1}\left(A_{1}\right) \cdot m_{2}\left(A_{2}\right)+\Delta(A, \Theta),
$$

where $\Delta(A, \Theta)$ depends on the specific algorithm and $A_{1}, A_{2} \subseteq \Theta$. In Yager [16] and Sun et al. [17], $\Delta_{\text {Yager }}(A, \Theta)=k$, if $A=\Theta$; otherwise, it becomes zero, and $\Delta_{\text {Sun }}(A, \Theta)=$ $k \cdot e^{-k} \cdot\left(\left(m_{1}(A)+m_{2}(A)\right) / 2\right)$ when system has two evidence sources.

According to formulae (17) and (18), it is easy to see that the improved part changes from $\Delta(A, \Theta)$ in DS evidence theory to $\Delta\left(A, 2^{\Theta}\right)$ in PCR5, which demonstrates that PCR5 rule can get higher combination precision with extra and larger computational complexity.

In addition, the general description of the modified conflict evidences methods expressed in Section 1 is shown as (assuming that there are $n$ evidences)

$$
m(A)=\sum_{A_{i} \subseteq \Theta} m_{i}\left(A_{i}\right) \cdot w_{i}\left(A_{i}\right),
$$


where $w_{i}\left(A_{i}\right)$ denotes the weighted factor assigned to $m_{i}\left(A_{i}\right)$. In Murphy [20] and Deng et al. [35], $w_{i \text {-Murphy }}\left(A_{i}\right)=1 / n$, where $n$ is the evidence number, and $w_{i \text {-Deng }}\left(A_{i}\right)$ represents evidence credibility gotten from the introduction of Euclidean distance.

Through comparison between PCR5 and the modified conflict evidences method in formulae (17) and (19), it is easy to verify that, different from PCR5 rule, this kind of approach takes the ambient noises into consideration and handles conflicts from the perspective of evidence credibility, which is more proximate to actual applications.

From what was mentioned above, PCR5 rule in DS evidence theory can be classified into the improved DS combination rules with better validity and larger computational complexity, and the modified conflict evidences methods take factors like environmental noises and sensors precision into account, which have better ability of practical applications relative to PCR5 rule. Thus, in order to get accurate, effective, and fast target recognition performance in IR/MMW system, this paper chooses to combine two mainstream improved methods and proposes the modified DS evidence theory presented in the next section.

\section{The Modified DS Evidence Theory}

The modified DS evidence theory proposed in this paper is designed to deal with the paradoxes caused by environmental uncertainty and imperfect knowledge and make the novel algorithm more suitable for IR/MMW target recognition system. It imitates the human brain's information reception processing and integration processing to realize information fusion in IR/MMW system. It firstly accurately assigns sensor priority through classifying evidences from different sources by their type and precision and calculates evidence credibility of each evidence through introduction of Minkowski distance, then modifies DS combination rule to avoid probable paradoxes caused by the normalization step, and, at last, uses decision-making rule to produce reliable synthesis results.

3.1. Discount Processing of Evidences. The discount processing of evidences is composed of two parts. One is the definition of source priority for distinguishing the differences of data obtained from different sensors according to their types and precision, and the other is the introduction of evidence credibility for analyses of different reliability of data from different sensors in consideration of weather and observation environment. The discount processing of evidences can decrease the conflicts caused by environmental uncertainty and differences among sensors, which is the precondition of DS combination rule.

3.1.1. Sensor Priority. Suppose that there are $M$ sensors with $N$ different types in IR/MMW system; we regard the data received from different sensors as the sources of evidence, whose number is also $M$ and type is $N$. Because the system has $N$ types of sensors, sensor priorities are also quantized into $N$ levels. According to the differences of sensor types and precision, the sensor priority can be defined to represent the dominance and importance of different sensors under certain identification mission. And for a particular environment of target recognition, sensor priorities of different sensors differ and satisfy $\operatorname{pr}\left(m_{i}\right) \in[0,1](i=1,2, \ldots, N)$.

This paper only assigns sensor priority by considering sensor types and precision to meet real-time demand of system; readers can take more factors into account by analogy. As sensor priorities are deemed to be the prior knowledge, assignment of evidence priorities depends on practical application. An example will make it clear: If there are five sensors in IR/MMW system with three same precision active IRs and two same precision passive MMWs, then these three active IRs must have the same sensor priorities, and two passive MMWs also have the same sensor priorities. The concrete value of sensor priority should be set by artificial operators based on system requirements. If system requires high spatial resolution and strong penetrating power, MMWs have obvious superiority and their sensor priorities should be higher than that of IRs, such as assignment as $\operatorname{pr}\left(m_{\mathrm{IRs}}\right)=0.9$, $\operatorname{pr}\left(m_{\mathrm{MMRs}}\right)=0.4$. On the contrary, if system demands great night work ability, sensor priorities of IRs will be much higher than that of MMWs.

3.1.2. Evidence Credibility. Sensor priority takes inequality and inconsistence caused by different types and precision of sensors into account, but uncertainty caused by factors like weather, observation environment, and missile-target distance is still the obstacle of obtaining accurate synthesis results. In order to relieve uncertainty and imperfection among evidences, evidence credibility is put forward to evaluate the reliability of different sensors before evidence combines.

Primarily, Minkowski distance is introduced as a distance function between two evidences. Assume that there are $N$ evidence sources, and $m_{i}$ and $m_{j}(i, j=1,2, \ldots, N)$ are two BPAs of mutually exclusive and exhaustive FoD: $\Theta=$ $\left\{A_{1}, A_{2}, \ldots, A_{M}\right\}$, which are, respectively, shown as

$$
\begin{aligned}
& m_{i}\left(A_{1}\right)=p_{1}, \\
& m_{i}\left(A_{2}\right)=p_{2}, \ldots, m_{i}\left(A_{M}\right)=p_{M} \\
& m_{j}\left(A_{1}\right)=q_{1}, \\
& m_{j}\left(A_{2}\right)=q_{2}, \ldots, m_{j}\left(A_{M}\right)=q_{M} .
\end{aligned}
$$

Minkowski distance is a distance measurement in European space, and it is an extension of Euclidean distance and Manhattan distance. According to the introduction of Minkowski distance, the distance function of $m_{i}$ and $m_{j}$ can be calculated as

$$
d_{i j}=d\left(m_{i}, m_{j}\right)=\left(\sum_{l=1}^{M}\left|p_{l}-q_{l}\right|^{m}\right)^{1 / m},
$$

where $m$ is a variable parameter. By definition, Minkowski distance will degrade into Manhattan distance when $m=1$, Euclidean distance when $m=2$, and Chebyshev distance when $m \rightarrow \infty$. In this paper, $m$ is unified to be 2 for the experimental convenience in Section 5 . 
Thus, the distance matrix is defined as

$$
\mathrm{DM}=\left[\begin{array}{cccc}
0 & d_{12} & \cdots & d_{1 N} \\
d_{21} & 0 & \cdots & d_{2 N} \\
\vdots & \vdots & \ddots & \vdots \\
d_{N 1} & d_{N 2} & \cdots & 0
\end{array}\right] .
$$

Then, the similarity function between two evidences is introduced as

$$
s_{i j}=s\left(m_{i}, m_{j}\right)=1-d_{i j} .
$$

It is obvious that the similarity function between two evidences will increase as the distance function decreases.

The similarity matrix of evidences is defined in the same way as shown before:

$$
\mathrm{SM}=\left[\begin{array}{cccc}
1 & s_{12} & \cdots & s_{1 N} \\
s_{21} & 1 & \cdots & s_{2 N} \\
\vdots & \vdots & \ddots & \vdots \\
s_{N 1} & s_{N 2} & \cdots & 1
\end{array}\right]
$$

Further, the support measurement and credibility of evidence $m_{i}$ are calculated separately as

$$
\begin{aligned}
& \sup \left(m_{i}\right)=\sum_{j=1, j \neq i}^{N} s\left(m_{i}, m_{j}\right) \\
& \operatorname{crd}\left(m_{i}\right)=\frac{\sup \left(m_{i}\right)}{\sum_{i=1}^{N} \sup \left(m_{i}\right)} .
\end{aligned}
$$

It can be seen from formula (26) that the credibility of evidences satisfies $\operatorname{crd}\left(m_{i}\right) \in[0,1]$ and $\sum_{i=1}^{N} \operatorname{crd}\left(m_{i}\right)=1$. The support measurement represents the similarity degree between certain evidence and other evidences, and credibility reflects the normalized support degree of certain evidence. It is clear that the support measurement and credibility of evidences will both increase when similarity function between certain evidence and other evidences increases.

3.1.3. Discount Processing. Assume sensor priority and evidence credibility for discount processing before evidences combine can enhance the consistency of sensors and reduce conflicts among evidences. The discount processing is defined as

$$
\begin{aligned}
& m^{\prime}(A)=\sum_{i=1}^{N} m_{i}(A) \cdot \operatorname{pr}\left(m_{i}\right) \cdot \operatorname{crd}\left(m_{i}\right) \\
& m^{\prime}(\Phi)=0 \quad \forall A \subseteq \Theta, A \neq \Phi .
\end{aligned}
$$

Because of $\operatorname{pr}\left(m_{i}\right) \in[0,1]$ and $\operatorname{crd}\left(m_{i}\right) \in[0,1]$, the discounted evidence satisfies $\sum_{A \subseteq \Theta} m^{\prime}(A) \leq 1$. To ensure the normalization of synthesis results, the discounted evidence should be normalized before evidences combine, which is defined as follows:

$$
m^{\prime \prime}(A)=\frac{m^{\prime}(A)}{\sum_{A \subseteq \Theta} m^{\prime}(A)} \quad A \subseteq \Theta .
$$

3.2. Improvement of DS Combination Rule. In the DS combination rule, paradoxes are mainly caused by incomplete FoD and the normalization step under the hypothesis of reliable evidences. In this section, paradoxes are only caused by the normalization step through assuming a complete FoD. Evidence conflicts have been greatly reduced by the discount processing with the introduction of sensor priority and evidence credibility. Under this circumstance, traditional DS combination rule can simply deal with common conflicts. However, conflicts caused by imperfect knowledge are still an urgent problem. In order to handle four common paradoxes described in Section 2, DS combination rule is improved correspondingly. The improvement of DS combination rule proportionally assigns global conflicts to propositions based on the discounted evidence instead of blindly negating information that hides in conflict evidences, which enhances the reliability and rationality of synthesis results.

The improved DS combination rule is defined as

$$
\begin{aligned}
& m(A)=\sum_{A_{1} \cap A_{2}=A} m^{\prime \prime}\left(A_{1}\right) \cdot m^{\prime \prime}\left(A_{2}\right)+k \cdot m^{\prime \prime}(A) \\
& m(\Phi)=0 \quad \forall A \subseteq \Theta, A \neq \Phi .
\end{aligned}
$$

Here, we need to further explain one point. The reason why it adopts assigning global conflicts to all propositions instead of assigning local conflicts directly to local propositions is that, without discount processing, local assignment method is more rational than global assignment, but in the current situation with discount processing, evidence conflicts have been drastically decreased. Global assignment method will lead to better convergence and less computation.

3.3. Decision-Making Rule. In DS evidence theory, two common decision-making rules are maximum belief function and maximum plausibility function method. But it is obvious that both of them are too simple to obtain accurate and satisfactory recognition results in IR/MMW system. Therefore, a compound decision-making rule is applied. Assume that the FoD is $\Theta=\left\{A_{1}, A_{2}, \ldots, A_{M}\right\}$; that is, $M$ targets are existent in target recognition system. If the synthesis results satisfy

$$
\begin{aligned}
& m\left(A_{1}\right)=\max \left\{m\left(A_{i}\right), A_{i} \subseteq \Theta\right\} \\
& m\left(A_{2}\right)=\max \left\{m\left(A_{j}\right), A_{j} \subseteq \Theta, A_{j} \neq A_{1}\right\} \\
& m\left(A_{1}\right)-m\left(A_{2}\right) \geq \varepsilon_{1} \\
& m(\Theta) \geq \varepsilon_{2} \\
& m\left(A_{1}\right) \geq m(\Theta),
\end{aligned}
$$

the recognition result will be target $A_{1}$, where $\varepsilon_{1}$ and $\varepsilon_{2}$ are preset threshold values.

\section{Application in IR/MMW Target Recognition}

As Section 3 gives specific evolution thread of the novel algorithm, this section will present the implementation steps of its application in IR/MMW system. Combining different 


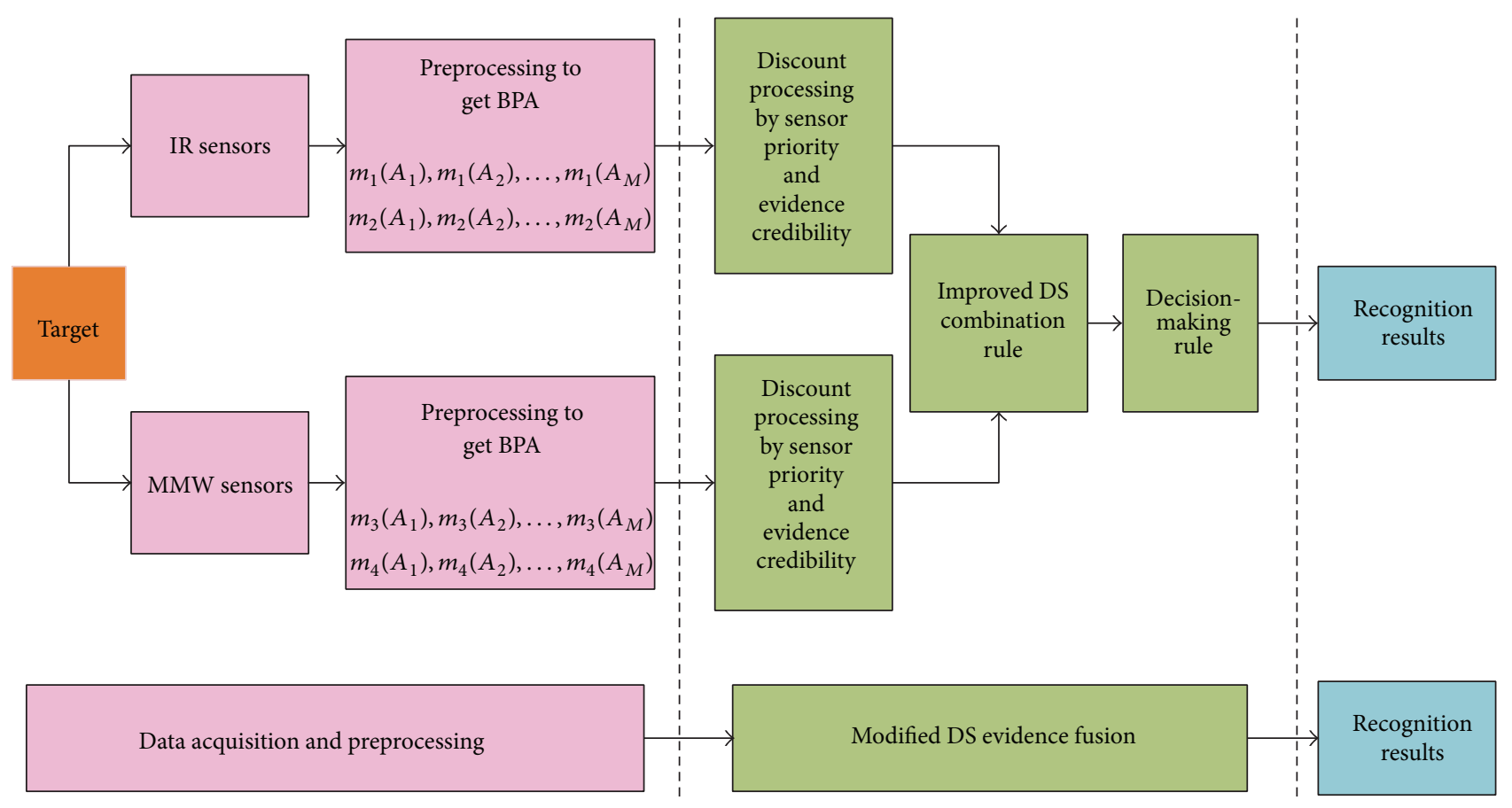

FIGURE 2: The flow diagram of IR/MMW system.

features of IRs and MMWs in target recognition system, the modified DS evidence theory is applied as a decision-making fusion method. Figure 2 shows the flow diagram of IR/MMW system (take an example of 2 IRs and 2 MMWs corresponding to experiments in Section 5).

Specific implementation steps of the application in IR/MMW system for target recognition are summarized as follows.

Step 1. Collect information of IRs and MMWs as evidences and assume that all sensors have the same FoD; obtain BPAs from all sensors through data acquisition and preprocessing. This step establishes a platform for information fusion.

Step 2. Utilize the modified DS evidence theory to combine evidences and make synthesis decision.

Step 3. Output the decision results as the recognition results.

\section{Experiment Results and Analyses}

In this section, we give two experiments. One is the comparison of performances between the modified algorithm and several existent methods, which proves its validity and reliability theoretically. The other is its application in IR/MMW system, which demonstrates its feasibility in practice.

5.1. Experiment 1: Theoretical Comparison between the Modified Algorithm and Existent Methods. Take four paradoxes described in Section 2 as examples to discuss the rationality and validity of the modified algorithm. The BPAs of four common paradoxes presented in Section 2.2 are shown in Table 1 and Figure 3.
TABLE 1: BPAs of four common paradoxes.

\begin{tabular}{lcccccc}
\hline \multirow{2}{*}{ Paradoxes } & Evidences & \multicolumn{5}{c}{ Propositions } \\
& & $A$ & $B$ & $C$ & $D$ & $E$ \\
\hline \multirow{3}{*}{ Complete conflict paradox } & $m_{1}$ & 1 & 0 & 0 & $\backslash$ & $\backslash$ \\
& $m_{2}$ & 0 & 1 & 0 & $\backslash$ & $\backslash$ \\
& $m_{3}$ & 0.8 & 0.1 & 0.1 & $\backslash$ & $\backslash$ \\
& $m_{4}$ & 0.8 & 0.1 & 0.1 & $\backslash$ & $\backslash$ \\
\hline \multirow{3}{*}{ trust paradox } & $m_{1}$ & 0.5 & 0.2 & 0.3 & $\backslash$ & $\backslash$ \\
& $m_{2}$ & 0.5 & 0.2 & 0.3 & $\backslash$ & $\backslash$ \\
& $m_{3}$ & 0 & 0.9 & 0.1 & $\backslash$ & $\backslash$ \\
1 trust paradox & $m_{4}$ & 0.5 & 0.2 & 0.3 & $\backslash$ & $\backslash$ \\
\hline & $m_{1}$ & 0.9 & 0.1 & 0 & $\backslash$ & $\backslash$ \\
& $m_{2}$ & 0 & 0.1 & 0.9 & $\backslash$ & $\backslash$ \\
& $m_{3}$ & 0.1 & 0.15 & 0.75 & $\backslash$ & $\backslash$ \\
High conflict paradox & $m_{4}$ & 0.1 & 0.15 & 0.75 & $\backslash$ & $\backslash$ \\
\hline & $m_{1}$ & 0.7 & 0.1 & 0.1 & 0 & 0.1 \\
& $m_{2}$ & 0 & 0.5 & 0.2 & 0.1 & 0.2 \\
& $m_{3}$ & 0.6 & 0.1 & 0.15 & 0 & 0.15 \\
& $m_{4}$ & 0.55 & 0.1 & 0.1 & 0.15 & 0.1 \\
& $m_{5}$ & 0.6 & 0.1 & 0.2 & 0 & 0.1 \\
\hline
\end{tabular}

It can be seen from Table 1 and Figure 3 that evidences in IR/MMW system can be divided into consistent evidences and conflict evidences. Apparently, the relatively consistent evidences are $m_{1}, m_{3}$, and $m_{4}$ in complete conflict paradox, $m_{1}, m_{2}$, and $m_{4}$ in 0 trust paradox, $m_{2}, m_{3}$, and $m_{4}$ in 1 trust paradox, and $m_{1}, m_{3}, m_{4}$, and $m_{5}$ in high conflict paradox. Thus, accurate synthesis results should agree with 

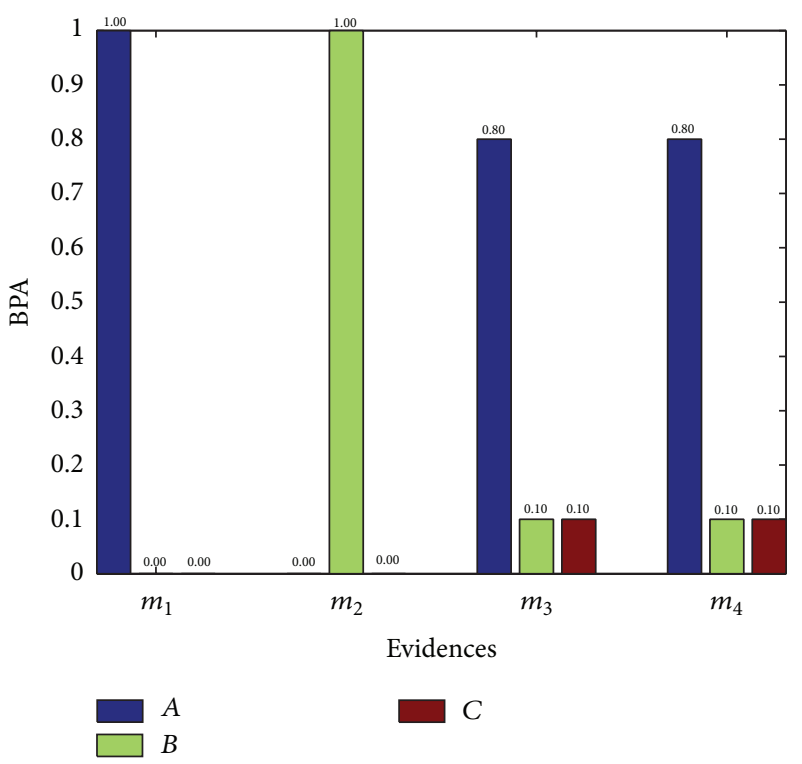

(a) BPA of complete conflict paradox
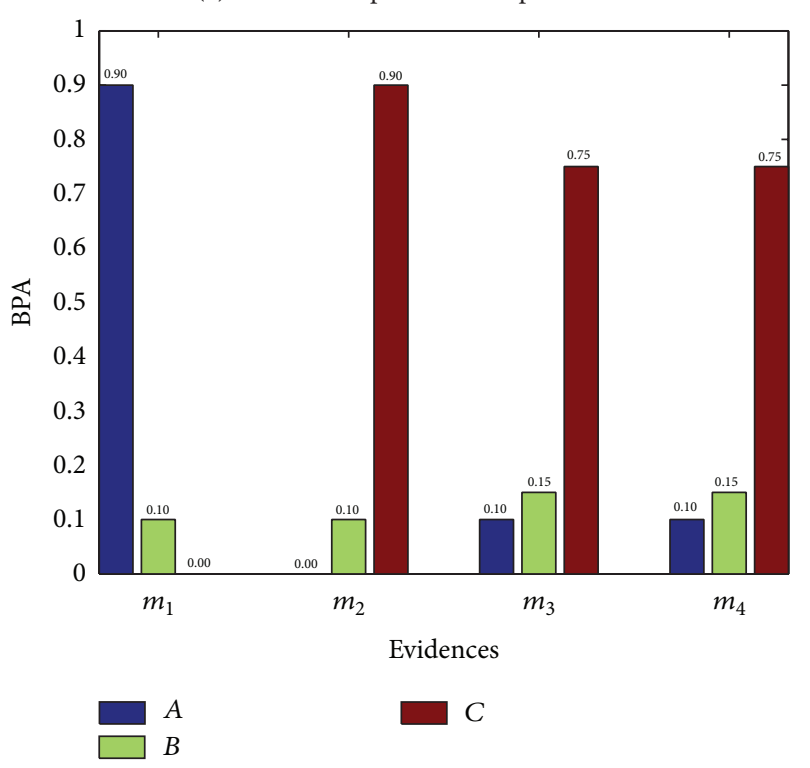

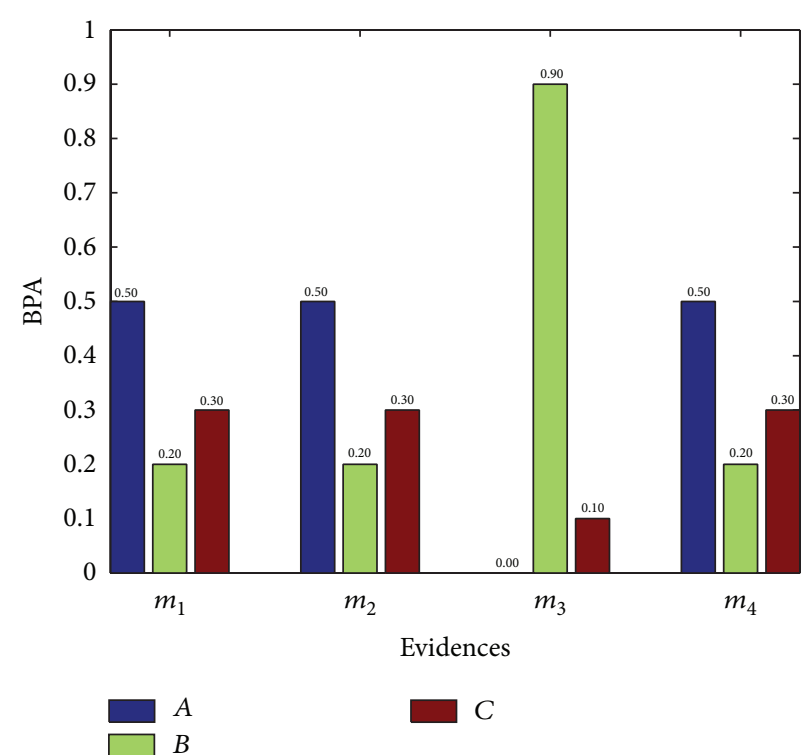

(b) BPA of 0 trust paradox
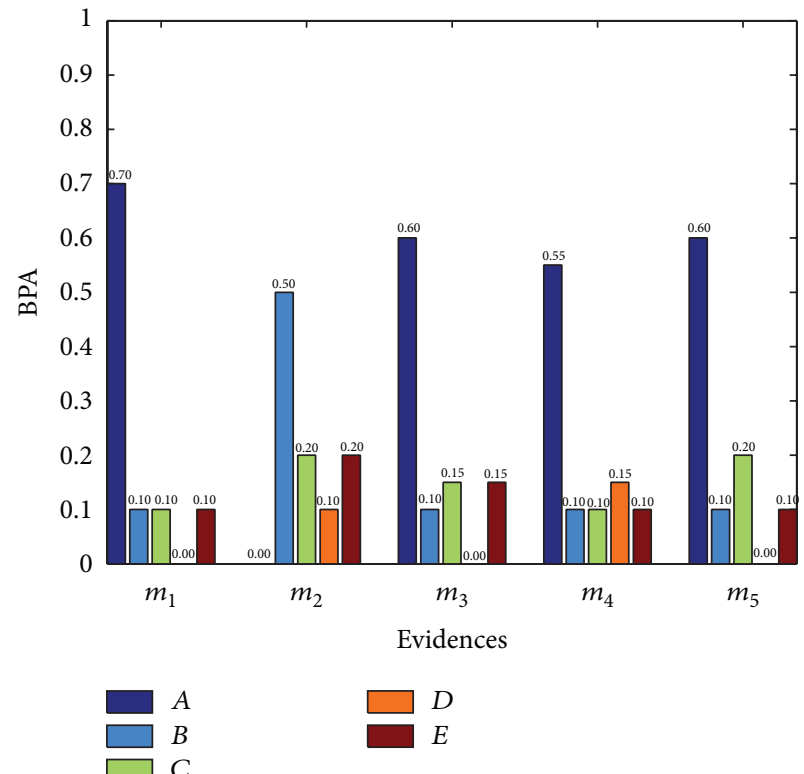

(d) BPA of high conflict paradox

(c) BPA of 1 trust paradox

Figure 3: BPAs of four common paradoxes.

these consistent evidences while being away from conflict evidences.

It is evident that traditional DS combination rule is unable to manage all four paradoxes, so we choose four existent improved methods, respectively, proposed by Yager [16], Sun et al. [17], Murphy [20], and Deng et al. [35] (called Yager, Sun, Murphy, and Deng for short) for comprehensive analyses with the modified method (called Modified for short). And the synthesis results are presented in Table 2 and Figure 4.

According to Table 2 and Figure 4, we make the following discussion.

5.1.1. In Complete Conflict Paradox $(k=1) . m_{1}, m_{3}$, and $m_{4}$ are the relatively consistent evidences. Yager gives $\Theta$ the whole belief as $m(\Theta)=1$, which, on the contrary, increases propositions' uncertainty. Sun only solves part of the conflicts as the BPAs of propositions $A, B$, and $C$ match the BPAs' proportion of these consistent evidences, but the BPA for $\Theta$ is still high as $m(\Theta)=0.8589$, which still has high uncertainty. At the same time, Murphy, Deng, and Modified can all get relatively rational results in complete conflict paradox.

5.1.2. In 0 Trust Paradox. $m_{1}, m_{2}$, and $m_{4}$ are the relatively consistent evidences. It is easy to check that the consistent evidences are the same, so the most valid algorithm should have the minimum difference between the synthesis results and the consistent evidences. As can be seen intuitively, Yager 
TABLE 2: Comparison of the synthesis results.

\begin{tabular}{|c|c|c|c|c|c|c|c|}
\hline \multirow{2}{*}{ Paradoxes } & \multirow{2}{*}{ Methods } & \multicolumn{6}{|c|}{ Propositions } \\
\hline & & $A$ & $B$ & C & $D$ & $E$ & $\Theta$ \\
\hline \multirow{5}{*}{ Complete conflict paradox $(k=1)$} & Yager & 0 & 0 & 0 & 1 & 1 & 1 \\
\hline & Sun & 0.0917 & 0.0423 & 0.0071 & 1 & 1 & 0.8589 \\
\hline & Murphy & 0.8204 & 0.1748 & 0.0048 & 1 & 1 & 0 \\
\hline & Deng & 0.8166 & 0.1164 & 0.0670 & 1 & 1 & 0 \\
\hline & Modified & 0.9242 & 0.0502 & 0.0256 & 1 & 1 & 0 \\
\hline \multirow{5}{*}{0 trust paradox $(k=0.99)$} & Yager & 0 & 0.7273 & 0.2727 & 1 & 1 & 0 \\
\hline & Sun & 0.0525 & 0.0597 & 0.0377 & 1 & 1 & 0.8501 \\
\hline & Murphy & 0.4091 & 0.4091 & 0.1818 & 1 & 1 & 0 \\
\hline & Deng & 0.4318 & 0.2955 & 0.2727 & 1 & 1 & 0 \\
\hline & Modified & 0.4679 & 0.2800 & 0.2521 & 1 & 1 & 0 \\
\hline \multirow{5}{*}{1 trust paradox $(k=0.9998)$} & Yager & 0 & 1 & 0 & 1 & 1 & 0 \\
\hline & Sun & 0.0388 & 0.0179 & 0.0846 & 1 & 1 & 0.8587 \\
\hline & Murphy & 0.1676 & 0.0346 & 0.7978 & 1 & 1 & 0 \\
\hline & Deng & 0.1388 & 0.1318 & 0.7294 & 1 & 1 & 0 \\
\hline & Modified & 0.0791 & 0.0743 & 0.8466 & 1 & 1 & 0 \\
\hline \multirow{5}{*}{ High conflict paradox $(k=0.9999)$} & Yager & 0 & 0.3571 & 0.4286 & 0 & 0.2143 & 0 \\
\hline & Sun & 0.0443 & 0.0163 & 0.0136 & 0.0045 & 0.0118 & 0.9094 \\
\hline & Murphy & 0.7637 & 0.1031 & 0.0716 & 0.0080 & 0.0538 & 0 \\
\hline & Deng & 0.5324 & 0.1521 & 0.1462 & 0.0451 & 0.1241 & 0 \\
\hline & Modified & 0.6320 & 0.1227 & 0.1171 & 0.0316 & 0.0967 & 0 \\
\hline
\end{tabular}

presents totally wrong results, and Modified is the most effective algorithm in this kind of paradox.

5.1.3. In 1 Trust Paradox. $m_{2}, m_{3}$, and $m_{4}$ are the relatively consistent evidences. Yager and Sun cannot handle this paradox practically which can be proved in similar way as shown in the discussion of complete conflict paradox. And Murphy, Deng, and Modified can manage 1 trust paradox in different degrees.

5.1.4. In High Conflict Paradox. $m_{1}, m_{3}, m_{4}$, and $m_{5}$ are the relatively consistent evidences. Yager and Sun are logical theoretically but cannot be put into practice because of their increasing uncertainty. And Murphy, Deng, and Modified all produce relatively reasonable results in this kind of paradox.

It is verified that Yager always produces wrong synthesis results under the condition of paradoxes, and it is unable to handle any kind of paradox. Sun allots most of the conflicts directly to $\Theta$, which just solves paradoxes theoretically. It is not suitable for practical application because of the increasing uncertainty of synthesis results. Murphy averages all evidences without separating consistent evidences and conflict evidences. And it is clear that it cannot solve paradoxes fundamentally because evidences' contributions to the synthesis results are totally different, in spite of its low computation. Therefore, only Deng and Modified can generate relatively reasonable synthesis results for all these four common paradoxes.

Then, the comparison of Deng and Modified is discussed here in detail. The evaluation criterion is composed of two parts: the weight distance measurement (wd) between the
TABLE 3: Comparison between Deng and Modified.

\begin{tabular}{lccc}
\hline Paradoxes & Methods & wd & $\nabla_{\mathrm{wd}}$ \\
\hline $\begin{array}{l}\text { Complete } \\
\text { conflict paradox }\end{array}$ & Deng & 0.1064 & $11.69 \%$ \\
\hline 0 trust paradox & Modified & 0.0940 & \\
\hline \multirow{2}{*}{1 trust paradox } & Deng & 0.1472 & $78.69 \%$ \\
\hline Complete & Deng & 0.1275 & \multirow{2}{*}{$20.37 \%$} \\
conflict paradox & Modified & 0.1015 & \\
\hline
\end{tabular}

synthesis result and original evidences and the decrement $\left(\nabla_{\mathrm{wd}}\right)$ between Deng and Modified, which are defined as

$$
\begin{aligned}
\mathrm{wd} & =\sum_{i=1}^{N} d\left(m_{i}, m\right) \cdot \operatorname{crd}\left(m_{i}\right) \\
\nabla_{\mathrm{wd}} & =\frac{\left|\mathrm{wd}_{2}-\mathrm{wd}_{1}\right|}{\mathrm{wd}_{1}} \cdot 100 \%,
\end{aligned}
$$

where $m_{i}(i=1,2, \ldots, N)$ and $m$, respectively, represent $N$ original evidences and the synthesis result and $\mathrm{wd}_{1}$ and $\mathrm{wd}_{2}$ separately represent the weight distance measurement of Deng and Modified.

In formula (31), we can see that when the synthesis result is consistent with original evidences, the weight distance measurement becomes small. This means that the smaller wd is, the more efficient this combination method is. The comparison is shown in Table 3. 


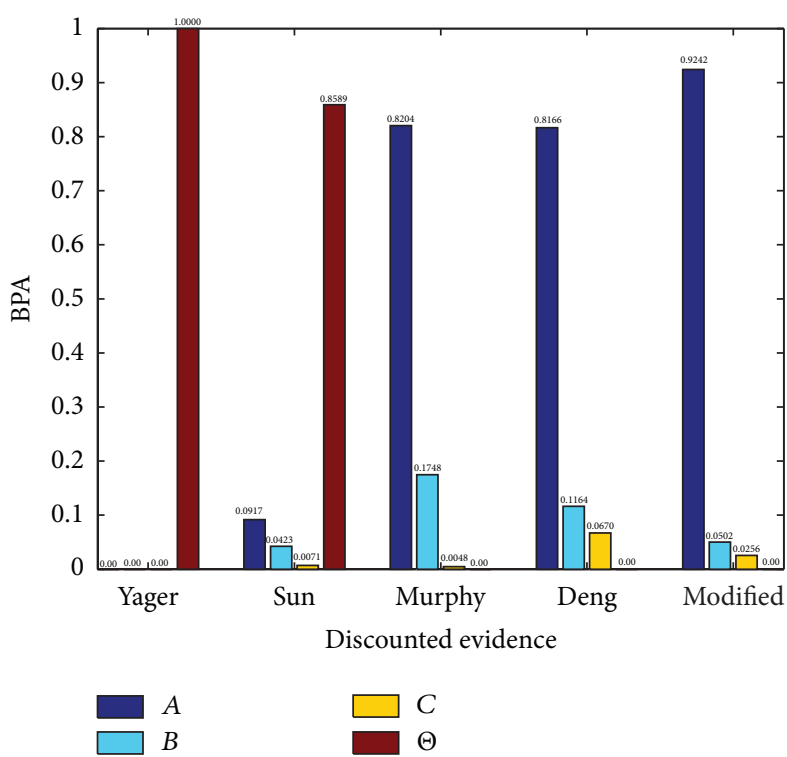

(a) Synthesis result of complete conflict paradox
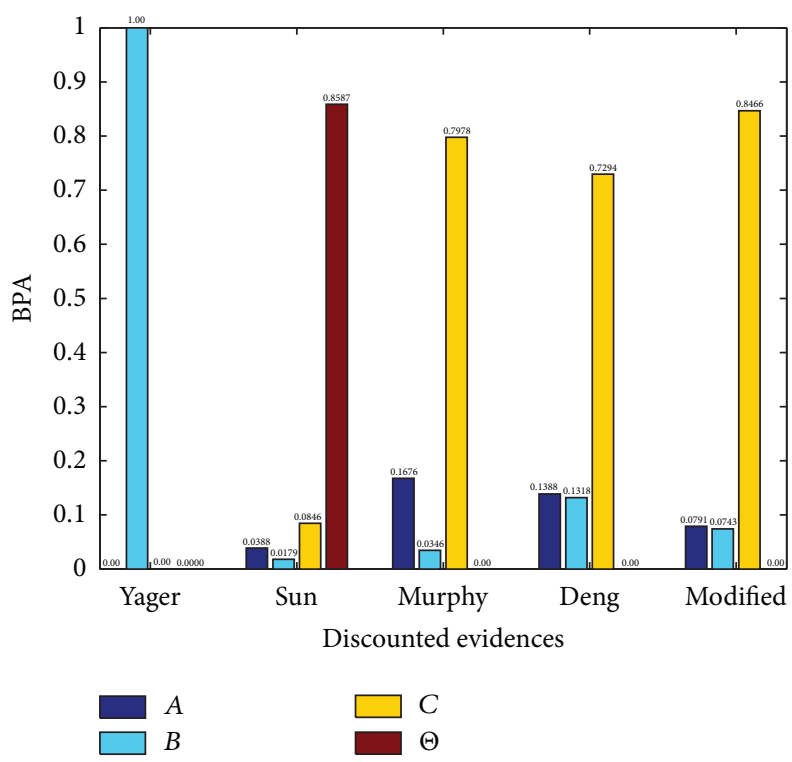

(c) Synthesis result of 1 trust paradox

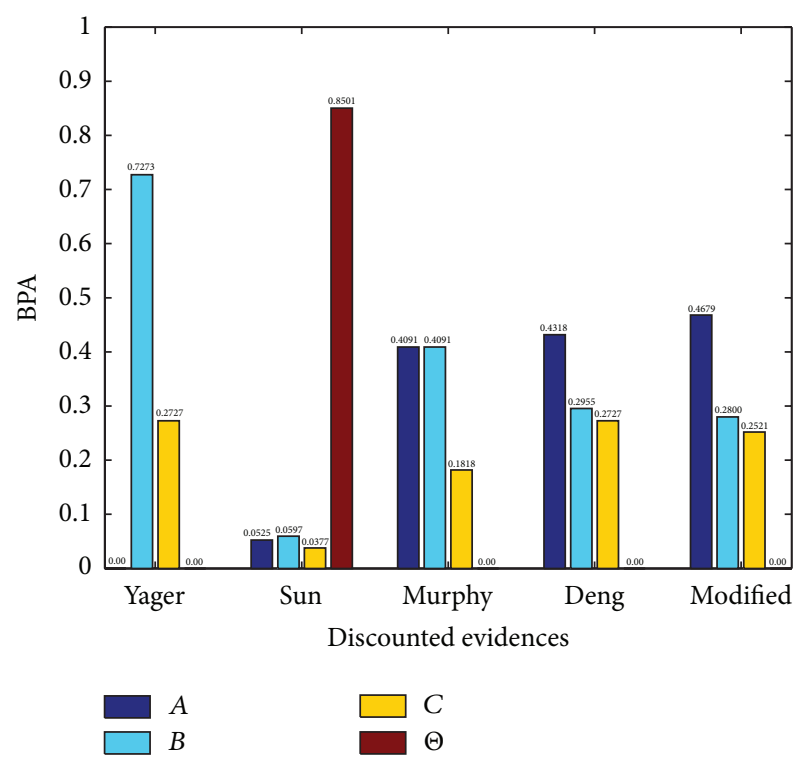

(b) Synthesis result of 0 trust paradox

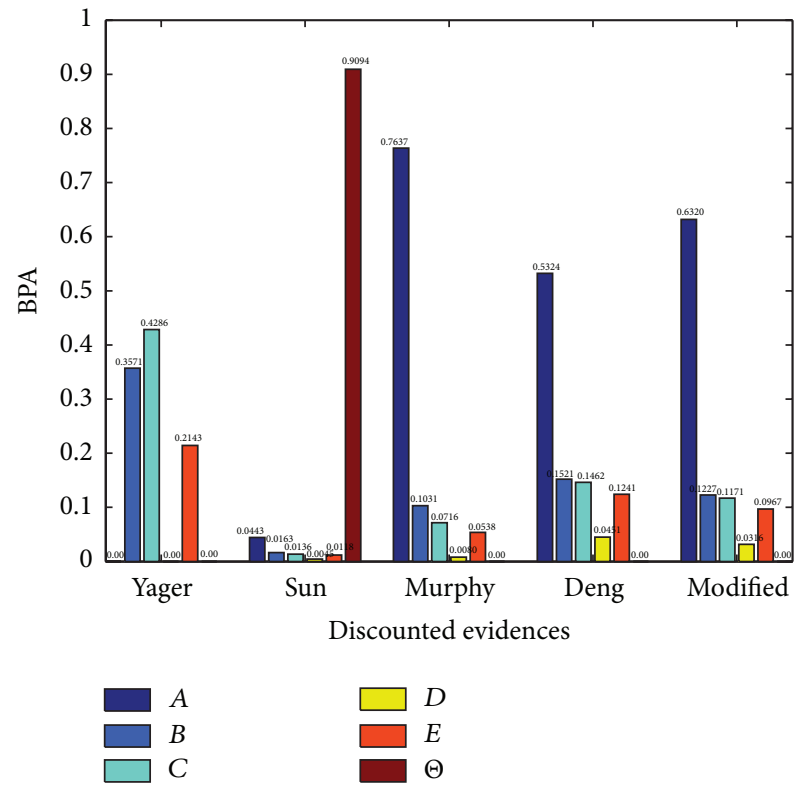

(d) Synthesis result of high conflict paradox

FIGURE 4: Comparison of the synthesis results.

It can be seen from Table 3 that wd of Modified is far less than that of Deng. It demonstrates that Modified can solve all the four paradoxes better, the evidences processed by Modified have better consistency and reliability, and the synthesis results produced by Modified are more rational and valid. Therefore, experiments here successfully certify theoretical rationality and feasibility of the modified DS evidence theory before its practical application.

There are two details needed to be illustrated. On the one hand, experiments here just discuss theoretical feasibility of the modified algorithm without considering its application; thus sensor priorities are all set to be 1 . This experiment represents the notion that every sensor is equally suitable for the current demands of system, while, in practical application, experiment results will be more effective and reliable with the introduction of sensor priority. On the other hand, in theoretical experiments, there is no need to prove the validity of decision-making rule, so it only outputs the synthesis results without decision-making. The advantages of sensor priority and decision-making rule are demonstrated in the next experiment.

\subsection{Experiment 2: Practical Experiment on Application of} $I R / M M W$ System. To illustrate practical application of the modified method, three groups of data are set here for target recognition, including two ordinary pieces of data (data 1 and 
TABLE 4: Recognition results of IR/MMW system.

\begin{tabular}{|c|c|c|c|c|c|c|c|}
\hline \multirow{2}{*}{ Targets } & \multicolumn{2}{|c|}{ Sensors } & \multicolumn{4}{|c|}{$m_{i}\left(A_{j}\right), i=1,2,3,4, A_{j} \subseteq \Theta$} & \multirow{2}{*}{ Recognition results } \\
\hline & Sensor type & Sensor priority & $A_{1}$ & $A_{2}$ & $A_{3}$ & $\Theta$ & \\
\hline \multirow{6}{*}{$A_{1}$} & IR1 & 0.53 & 0.25 & 0.15 & 0.20 & 0.40 & $\Theta$ \\
\hline & IR2 & 0.53 & 0.20 & 0.35 & 0.20 & 0.25 & $A_{2}$ \\
\hline & MMW1 & 0.85 & 0.60 & 0.15 & 0.10 & 0.15 & $A_{1}$ \\
\hline & MMW2 & 0.85 & 0.65 & 0.10 & 0.15 & 0.10 & $A_{1}$ \\
\hline & \multicolumn{2}{|c|}{ Discounted evidence } & 0.4759 & 0.1715 & 0.1526 & 0.2000 & $A_{1}$ \\
\hline & \multicolumn{2}{|c|}{ IR/MMW fusion } & 0.5885 & 0.1599 & 0.1394 & 0.1122 & $A_{1}$ \\
\hline \multirow{6}{*}{$A_{2}$} & IR1 & 0.86 & 0.10 & 0.60 & 0.10 & 0.20 & $A_{2}$ \\
\hline & IR2 & 0.86 & 0.10 & 0.70 & 0.10 & 0.10 & $A_{2}$ \\
\hline & MMW1 & 0.45 & 0.20 & 0.30 & 0.10 & 0.40 & $\Theta$ \\
\hline & MMW2 & 0.45 & 0.20 & 0.25 & 0.35 & 0.20 & $A_{3}$ \\
\hline & \multicolumn{2}{|c|}{ Discounted evidence } & 0.1330 & 0.5251 & 0.1407 & 0.2012 & $A_{2}$ \\
\hline & \multicolumn{2}{|c|}{ IR/MMW fusion } & 0.1144 & 0.6576 & 0.1221 & 0.1059 & $A_{2}$ \\
\hline \multirow{6}{*}{$A_{3}$} & IR1 & 0.40 & 0.12 & 0.21 & 0.32 & 0.35 & $\Theta$ \\
\hline & IR2 & 0.35 & 0.35 & 0.06 & 0.29 & 0.30 & $A_{1}$ \\
\hline & MMW1 & 0.60 & 0.29 & 0.03 & 0.28 & 0.40 & $\Theta$ \\
\hline & MMW2 & 0.65 & 0.05 & 0.20 & 0.43 & 0.32 & $A_{3}$ \\
\hline & \multicolumn{2}{|c|}{ Discounted evidence } & 0.1894 & 0.1261 & 0.3376 & 0.3469 & $\Theta$ \\
\hline & \multicolumn{2}{|c|}{ IR/MMW fusion } & 0.2167 & 0.1363 & 0.4362 & 0.2108 & $A_{3}$ \\
\hline
\end{tabular}

data 2) and one adverse piece of data (data 3). According to the data acquisition and preprocessing method described in [36], we extract the features of information from IRs and MMWs and then use grey correlation classifier to get BPAs as the input of the modified DS evidence fusion.

Experiment conditions are as follows: in IR/MMW system, assume that the FoD is $\Theta=\left\{A_{1}, A_{2}, A_{3}\right\}$, where $A_{1}, A_{2}$, and $A_{3}$ separately represent reconnaissance aircraft, bomber aircraft, and fighters. IRs and MMWs provide information about targets as evidences, and there are four evidence sources including IR1, IR2, MMW1, and MMW2. The sensor priorities are expressed as $\operatorname{pr}\left(m_{1}\right), \operatorname{pr}\left(m_{2}\right), \operatorname{pr}\left(m_{3}\right)$, and $\operatorname{pr}\left(m_{4}\right)$, and the preset threshold values in decision-making rule are $\varepsilon_{1}=0.2, \varepsilon_{2}=0.25$.

Data 1 (Target Recognition for $A_{1}$ ). Assume that there is a lot of smoke, fog, and cloud in observation environment (actual battlefield). And system requires high spatial resolution and strong penetrating power. Under these conditions, MMWs have obvious advantage over IRs. As prior knowledge, sensor priorities are set as $\operatorname{pr}\left(m_{1}\right)=\operatorname{pr}\left(m_{2}\right)=0.53, \operatorname{pr}\left(m_{3}\right)=$ $\operatorname{pr}\left(m_{4}\right)=0.85$.

Data 2 (Target Recognition for $A_{2}$ ). Assume that target recognition mission is marched in the night and system requires great night work ability and strong concealment. It is obvious that IRs have obvious advantage over MMWs in this situation. As prior knowledge, sensor priorities are set as $\operatorname{pr}\left(m_{1}\right)=\operatorname{pr}\left(m_{2}\right)=0.86, \operatorname{pr}\left(m_{3}\right)=\operatorname{pr}\left(m_{4}\right)=0.45$

Data 3 (Target Recognition for $A_{3}$ ). Assume that observation environment is abominable due to environmental clutter and artificial interference. Single-mode system will not produce correct recognition results. In order to ensure the reliability of target recognition, the fusion of IRs and MMWs is an inevitable trend. If system requires wide range of search and interception, MMWs have obvious advantage over IRs. As prior knowledge, sensor priorities are set as $\operatorname{pr}\left(m_{1}\right)=0.40$, $\operatorname{pr}\left(m_{2}\right)=0.35, \operatorname{pr}\left(m_{3}\right)=0.60$, and $\operatorname{pr}\left(m_{4}\right)=0.65$.

According to the above data, the recognition results of IR/MMW system are presented in Table 4 and Figure 5.

It can be seen from Table 4 and Figure 5 that, in target recognition of $A_{1}$, system is led mainly by MMWs with complement by IRs according to their sensor priorities. In the single-mode systems, MMWs can identify objective correctly, while IRs cannot. And IR/MMW fusion can not only identify real objective, but also enhance the accuracy of recognition results by greatly increasing the BPA of real target. In similar principle when identifying $A_{2}$, the syncretic recognition results verify rationality and validity of the modified DS evidence theory in IR/MMW system. Experiment results of data 3 show that all sensor priorities are low because of poor observation environment, and only MMW2 in single-mode systems can identify real objective but with large BPA for $\Theta$. This demonstrates that large uncertainty exists in this singlemode's recognition results. However, under that experimental condition, recognition results in fusion system significantly decrease uncertainty of system to facilitate decision-making. In addition, the modified algorithm in IR/MMW system can still accurately identify objective, even under the condition that two or three of the sensors are unable to identify real objective, and greatly increase reliability of IR/MMW system.

Through the above analyses, application of the modified DS evidence theory in IR/MMW system can efficiently fuse multisensor information, accurately identify objective, and 


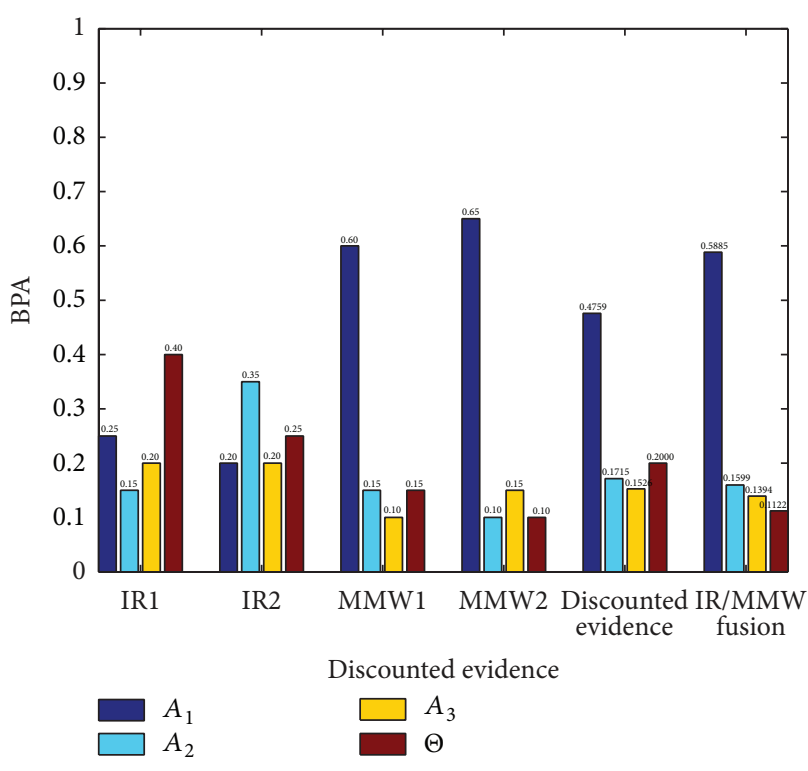

(a) Target recognition for $A_{1}$

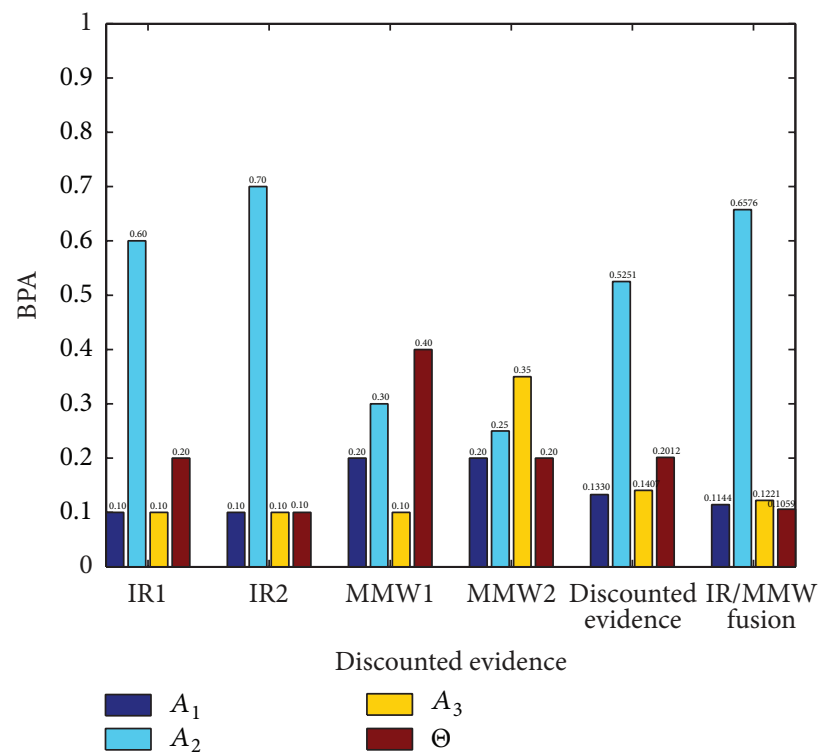

(b) Target recognition for $A_{2}$

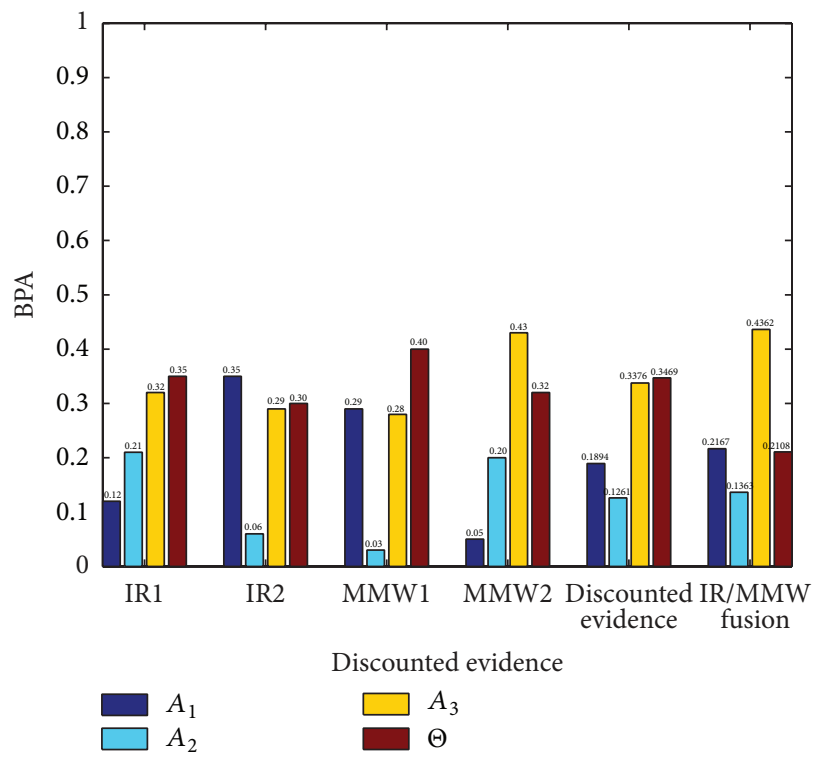

(c) Target recognition for $A_{3}$

FIGURE 5: Recognition results of IR/MMW system.

improve recognition rate and system reliability. It directly proves the validity and rationality of the modified algorithm and adaptability and flexibility of the system.

\section{Conclusion}

This paper proposes a modified DS evidence theory method for target recognition in IR/MMW system. With the discount processing and modified DS combination rule, the novel algorithm theoretically solves paradoxes caused by inconsistency and inequality of evidences, reduces the negative effects caused by complex environmental factors, and improves effectiveness and accuracy of the synthesis results. And the introduction of the compound decision-making rule further ensures producing satisfactory recognition results. Experiment results and analyses demonstrate that application of the modified algorithm as an information fusion method in IR/MMW system can enhance recognition rate of system and improve the accuracy and antijamming immunity of guidance system. Thus, it greatly enhances the operational performance of weapon system under various environmental conditions and realizes the all-weather operations. That is, it has great engineering application value.

In the further study, there are two technical researches that need to be studied. With the increasing number of sensors, there will be a huge computation burden for system along with great recognition performance. And the application of DSmT in target recognition system should be studied 
to realize the uncertainty reasoning better. Therefore, this method should be simplified and optimized.

\section{Conflict of Interests}

The authors declare that there is no conflict of interests regarding the publication of this paper.

\section{Acknowledgments}

This work is supported by the National Natural Science Foundation of China (no. 61301095, no. 51374099), the Heilongjiang Province Natural Science Foundation (no. F201345), and the Fundamental Research Funds for the Central Universities of China (no. HEUCF150812/150810).

\section{References}

[1] A. P. Dempster, "Upper and lower probabilities induced by a multivalued mapping," Annals of Mathematical Statistics, vol. 38, pp. 325-339, 1967.

[2] G. Shafer, A Mathematical Theory of Evidence, Princeton University Press, Princeton, NJ, USA, 1976.

[3] Y. Deng and F. T. S. Chan, "A new fuzzy dempster MCDM method and its application in supplier selection," Expert Systems with Applications, vol. 38, no. 8, pp. 9854-9861, 2011.

[4] M. Beynon, D. Cosker, and D. Marshall, "An expert system for multi-criteria decision making using Dempster Shafer theory," Expert Systems with Applications, vol. 20, no. 4, pp. 357-367, 2001.

[5] X. Y. Su, Y. Deng, S. Mahadevan, and Q. L. Bao, "An improved method for risk evaluation in failure modes and effects analysis of aircraft engine rotor blades," Engineering Failure Analysis, vol. 26, pp. 164-174, 2012.

[6] C. R. Parikh, M. J. Pont, and N. B. Jones, "Application of Dempster-Shafer theory in condition monitoring applications: a case study," Pattern Recognition Letters, vol. 22, no. 6-7, pp. 777-785, 2001.

[7] Z. Dou, X. C. Xu, Y. Lin, and R. L. Zhou, "Application of D$S$ evidence fusion method in the fault detection of temperature sensor," Mathematical Problems in Engineering, vol. 2014, Article ID 395057, 6 pages, 2014.

[8] X. Fan and M. J. Zuo, "Fault diagnosis of machines based on D-S evidence theory. Part 1: D-S evidence theory and its improvement," Pattern Recognition Letters, vol. 27, no. 5, pp. 366-376, 2006.

[9] Y. Hu, X. Fan, H. Zhao, and B. Hu, "The research of target identification based on neural network and D-S evidence theory," in Proceedings of the International Asia Conference on Informatics in Control, Automation and Robotics (CAR '09), pp. 345-349, Bangkok, Thailand, Feburary 2009.

[10] G. Dong and G. Kuang, "Target recognition via information aggregation through Dempster-Shafer's evidence theory," IEEE Geoscience and Remote Sensing Letters, vol. 12, no. 6, pp. 12471251, 2015.

[11] J. Dezert, "Autonomous navigation with uncertain reference points using the PDAF," in Multitarget-Multisensor Tracking, Y. Bar-Shalom, Ed., vol. 2, pp. 271-324, Artech House, 1991.

[12] L. Dymova and P. Sevastjanov, "An interpretation of intuitionistic fuzzy sets in terms of evidence theory: decision making aspect," Knowledge-Based Systems, vol. 23, no. 8, pp. 772-782, 2010.

[13] J. Kang, Y.-B. Gu, and Y.-B. Li, "Multi-sensor information fusion algorithm based on DS evidence theory," Journal of Chinese Inertial Technology, vol. 20, no. 6, pp. 670-673, 2012.

[14] X. X. Wang and F. B. Yang, Research on the combination rule of conflict evidences [Ph.D. thesis], North University of China, Taiyuan, China, 2007.

[15] P. Smets, "The combination of evidence in the transferable belief model," IEEE Transactions on Pattern Analysis and Machine Intelligence, vol. 12, no. 5, pp. 447-458, 1990.

[16] R. R. Yager, "On the aggregation of prioritized belief structures," IEEE Transactions on Systems, Man, and Cybernetics-Part A: Systems and Humans, vol. 26, no. 6, pp. 708-717, 1996.

[17] Q. Sun, X. Q. Ye, and W. K. Gu, "A new combination rules of evidence theory," Chinese Journal of Electronics, vol. 28, no. 8, pp. 117-119, 2000.

[18] E. Lefevre, O. Colot, P. Vannoorenberghe, and D. De Brucq, "A generic framework for resolving the conflict in the combination of belief structures," in Proceedings of the 3rd International Conference on Information Fusion (FUSION '00), vol. 1, pp. MoD411-MoD418, Paris, France, July 2000.

[19] Q. Pan, S. Y. Zhang, and H. C. Zhang, "A new kind of combination rule of evidence theory," Control and Decision, vol. 15, no. 5, pp. 540-544, 2000.

[20] C. K. Murphy, "Combining belief functions when evidence conflicts," Decision Support Systems, vol. 29, no. 1, pp. 1-9, 2000.

[21] T. Horiuchi, "Decision rule for pattern classification by integrating interval feature values," IEEE Transactions on Pattern Analysis and Machine Intelligence, vol. 20, no. 4, pp. 440-448, 1998.

[22] H. W. Guo, K. W. Shi, Q. K. Lin, and Y. Deng, "A new combination rule of evidence," Journal of Shanghai Jiao-Tong University, vol. 40, no. 11, pp. 1895-1900, 2007.

[23] E. Blasch, Derivation of a belief filter for simultaneous high range resolution radar tracking and identification [Ph.D. thesis], Wright State University, Dayton, Ohio, USA, 1999.

[24] E. Blasch and B. Kahler, "Multiresolution EO/IR target tracking and identification," in Proeedings of the 8th International Conference on Information Fusion, vol. 1, pp. 275-282, July 2005.

[25] L. J. Xu, Y. Z. Chen, and P. Y. Cui, "Improvement of D-S evidential theory in multisensor data fusion system," in Proceedings of the 5th World Congress on Intelligent Control and Automation (WCICA '04), vol. 4, pp. 3124-3128, June 2004.

[26] X.-M. Zhang, J.-Q. Han, and X.-B. Xu, "Dempster-Shafer reasoning with application multisensor object recognition system," in Proceedings of the International Conference on Machine Learning and Cybernetics, vol. 2, pp. 975-977, IEEE, Shanghai, China, August 2004.

[27] A. Martin and E. Radoi, "Effective ATR algorithms using information fusion models," in Proceedings of the 7th International Conference on Information Fusion, vol. 28, Stockholm, Sweden, June-July 2004.

[28] E. Blasch, A. Josang, J. Dezert, P. C. G. Costa, K. B. Laskey, and A.-L. Jousselme, "URREF self-confidence in information fusion trust," in Proceedings of the IEEE 17th International Conference on Information Fusion (FUSION '14), pp. 1-8, Salamanca, Spain, July 2014.

[29] F. Smarandache and J. Dezert, "An introduction to DSm theory of plausible, paradoxist, uncertain, and imprecise reasoning for information fusion," Octogon Mathematical Magazine, vol. 15, no. 2, pp. 681-722, 2007. 
[30] J. Dezert, Z.-G. Liu, and G. Mercier, "Edge detection in color images based on DSmT," in Proceedings of the 14th International Conference on Information Fusion (FUSION '11), pp. 1-8, IEEE, Chicago, Ill, USA, July 2011.

[31] J. Pan and J. Dezert, "Automatic aircraft recognition using DSmT and HMM," in Proceedings of the 17th International Conference on Information Fusion (FUSION '14), Salamanca, Spain, July 2014.

[32] F. Haouas and Z. Ben Dhiaf, "New contributions into the Dezert-Smarandache theory: application to remote sensing image classification," in Proceedings of the 6th International Conference on Soft Computing and Pattern Recognition (SoCPaR '14), pp. 319-324, Tunis, Tunisia, August 2014.

[33] E. Blasch, J. Dezert, and B. Pannetier, "Overview of DempsterShafer and belief function tracking methods," in SPIE Defense, Security, and Sensing. International Society for Optics and Photonics, vol. 8745, May 2013.

[34] J. Dezert and F. Smarandache, Advances and Applications of DSmT for Information Fusion (Collected Works), vol. 1-4, American Research Press, 2004-2014.

[35] Y. Deng, W.-K. Shi, and Z.-F. Zhu, "Efficient combination approach of conflict evidence," Journal of Infrared and Millimeter Waves, vol. 23, no. 1, pp. 27-32, 2004.

[36] J. Guo, Research on the key technologies of multi-sensor fusion target recognition [M.S. thesis], National University of Defense Technology, Changsha, China, 2008. 


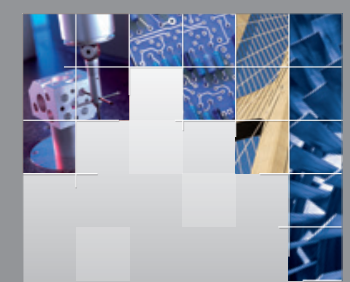

\section{Enfincering}
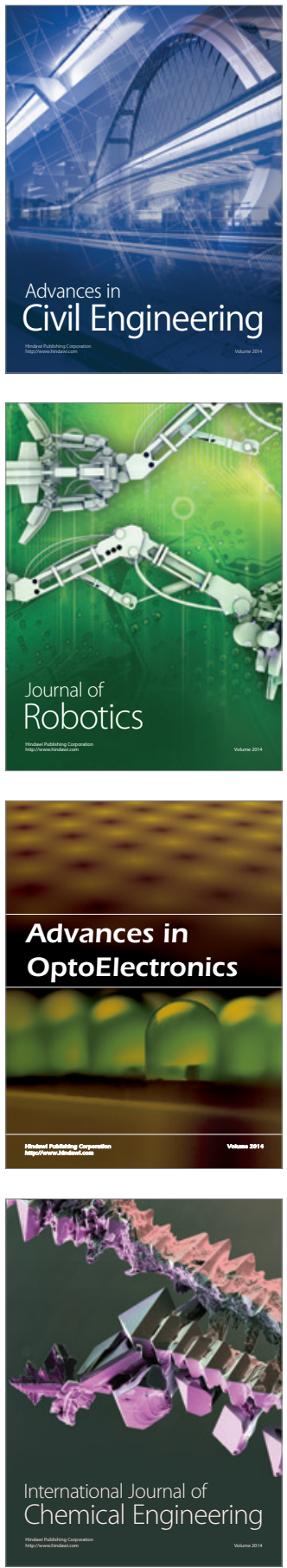

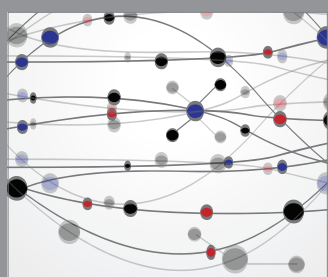

The Scientific World Journal

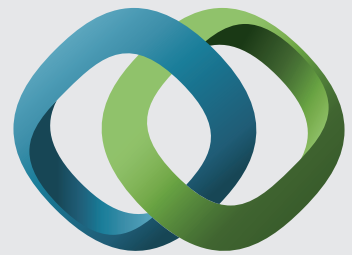

\section{Hindawi}

Submit your manuscripts at

http://www.hindawi.com
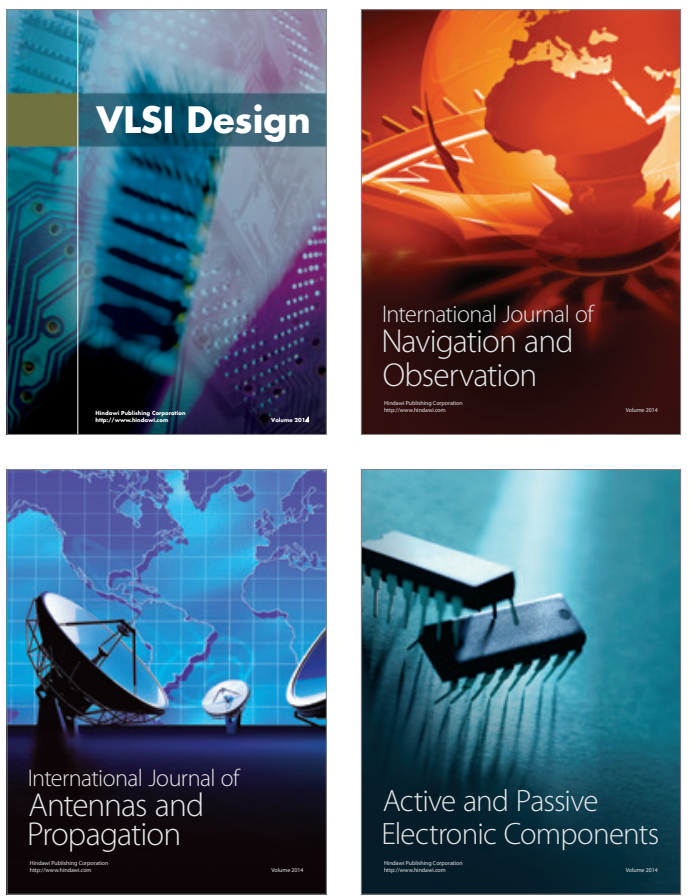
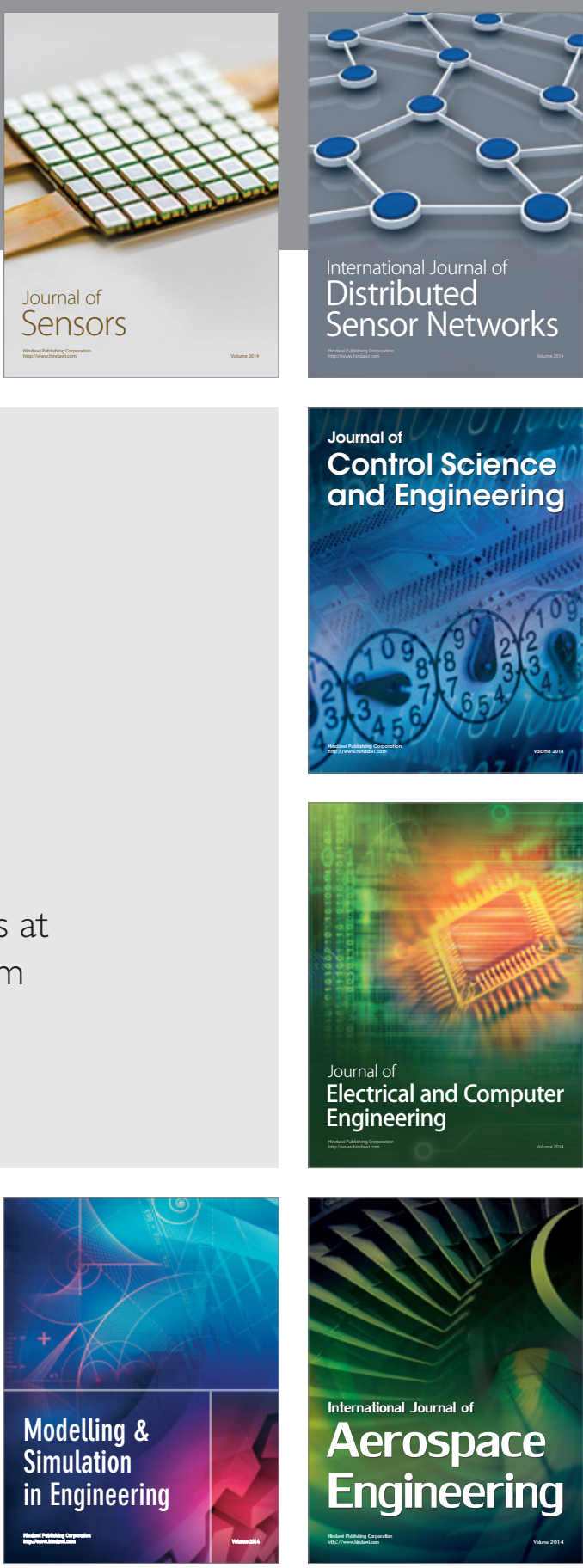

International Journal of

Distributed

Sensor Networks

Journal of

Control Science

and Engineering
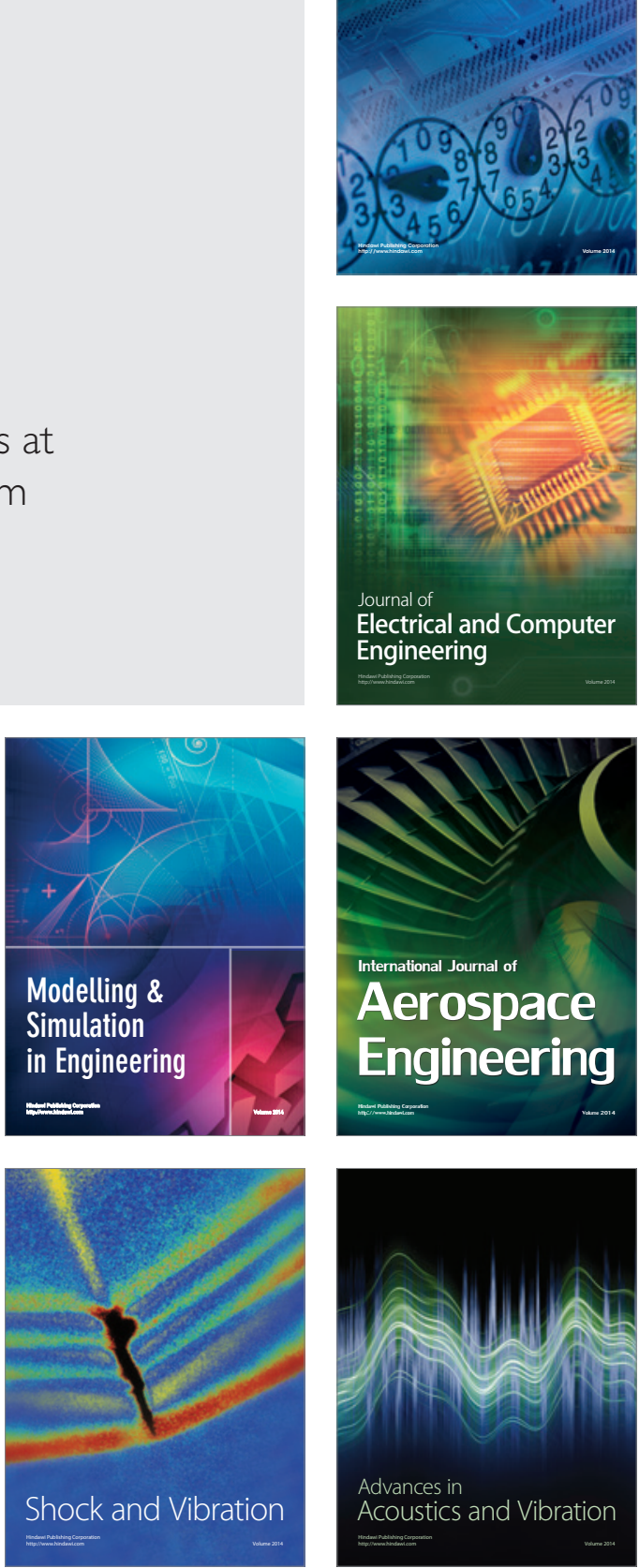